\title{
Recommending Missing and Suspicious Links in Multiplex Financial Networks
}

\author{
Robert E. Tillman, Prashant Reddy, Manuela Veloso \\ J.P. Morgan AI Research \\ New York, NY
}

\begin{abstract}
Many complex systems in finance can be modeled as multiplex networks, or networks which depict multiple types of interactions between entities. We consider the problem of detecting missing and suspicious interactions in multiplex financial networks in a real world context where predictions are provided continuously according to budget limitations. We propose a recommendation system based on a recently proposed heuristic for link prediction and incorporate feedback from previous recommendations to improve the system's performance over time. We provide theoretical conditions which show our approach approximates an (intractable) entropy-minimization solution while remaining computationally efficient and providing recommendations that are explainable. We apply our approach to a real world multiplex financial network and demonstrate its effectiveness at discovering missing and false links.
\end{abstract}

\section{KEYWORDS}

financial networks, link prediction, anomaly detection, recommendation systems, explainable predictions

\section{ACM Reference Format:}

Robert E. Tillman, Prashant Reddy, Manuela Veloso. 2020. Recommending Missing and Suspicious Links in Multiplex Financial Networks. In ACM International Conference on AI in Finance (ICAIF '20), October 15-16, 2020, New York, NY, USA. ACM, New York, NY, USA, 8 pages. https://doi.org/10. $1145 / 3383455.3422538$

\section{INTRODUCTION}

Many complex systems in finance can be efficiently represented using networks. Financial transactions can be represented as edges or links between nodes representing individuals, institutions or intermediaries. In previous work, networks have been used to model bilateral relationships and exposures of financial institutions to study contagion risk $[2,5,10]$ and measure the interconnectedness of the global financial system [9]. Networks have also been used in finance to model the organizational complexity of companies from financial statements [4], shareholders of publicly traded companies [20], boards of directors and mutual fund holdings structure [10].

Many such systems are multiplex in nature, or involve multiple types of interactions or relationships between entities. For example,

Permission to make digital or hard copies of all or part of this work for personal or classroom use is granted without fee provided that copies are not made or distributed for profit or commercial advantage and that copies bear this notice and the full citation on the first page. Copyrights for components of this work owned by others than ACM must be honored. Abstracting with credit is permitted. To copy otherwise, or republish, to post on servers or to redistribute to lists, requires prior specific permission and/or a fee. Request permissions from permissions@acm.org.

ICAIF '20, October 15-16, 2020, New York, NY, USA

(C) 2020 Association for Computing Machinery.

ACM ISBN 978-1-4503-7584-9/20/10 .. \$15.00

https://doi.org/10.1145/3383455.3422538 in networks which describe payments between individuals or institutions, we may observe $\mathrm{ACH}$ payments, wire transfers, mobile payments, etc. We may also be able to overlay such networks with additional information about entities' interactions from private deals, real estate records, holdings data or other information. A multiplex network [15] is a formal network model which can be used to represent these multiple types of interactions.

While networks have primarily been used in finance to model interactions between financial institutions and other market participants, they are also useful for modeling interactions within large financial institutions. Banks, broker/dealers, exchanges and other institutions consume enormous amounts data of different types which are processed, stored and accessed by a variety of different internal systems. Much of this data is highly sensitive and subject to strict regulatory and compliance requirements. Ensuring the integrity and security of this data across a multitude of systems and maintaining appropriate segregation of data between different lines of business is a complex task.

One of the most common network inference tasks is link prediction, or predicting possible links between network entities that are either missing or likely to form in the future. Anomalous link detection, or predicting which existing links are incorrect or likely to be removed in the future is a related task. There are numerous applications of these tasks in financial networks. Anomalous links in payment networks may correspond to fraudulent transactions. Missing links in such networks might correspond to concealed transactions which could be useful in anti-money laundering systems. In networks representing internal systems within financial institutions, link prediction and anomalous link detection can be used to detect and verify data usage between internal systems.

Numerous methods have been proposed for the link prediction problem in simple networks with only a single type of interaction between entities, including unsupervised heuristics based on assumptions from social network analysis, e.g. individuals with common connections are more likely to be connected [1, 3, 13, 16, 18, 22, 27], as well as supervised methods based on random walk embeddings $[12,21,25]$ and graph neural networks $[14,28]$. Several recent approaches consider extending link prediction to multiplex networks. [26] proposes a framework for extending unsupervised heuristics to the multiplex domain based on similarity between different types of interactions: a type of link is considered more likely if a similar link exists or is predicted between the same two nodes.

We consider link prediction and anomalous link detection in multiplex financial networks in a real world settings where predictions are provided continuously and there are budget constraints limiting how many predictions can be evaluated over a given interval. We pose this as a recommendation system problem where we iteratively recommend missing and suspicious links which are then 
evaluated with feedback, allowing the network to be updated to improve future recommendations. This is in contrast to the typical offline setting under which most link prediction methods are evaluated. We show that the CWC heuristic from [26] can be adapted to this setting to efficiently recommend both missing and anomalous links. We propose an approach which approximates the solution to an intractable entropy minimization formulation of the problem while remaining computationally efficient and providing explainable recommendations. Finally, we apply the approach to a real multiplex financial network and demonstrate its effectiveness at discovering missing and suspicious links.

Section 2 provides background on multiplex networks and link prediction. In sections 3 and 4, we propose a notion of entropy for multiplex networks and formalize the problem of recommending links as entropy minimization, which we then show can be approximated using an efficient greedy approach. We empirically evaluate our approach on a real multiplex financial network in section 5.

\section{MULTIPLEX NETWORK LINK PREDICTION}

Similarity heuristics are unsupervised approaches for link prediction based on graph topology and assumptions from social network analysis and other fields. Their advantages include simplicity, interpretability and computational efficiency. Despite their simplicity, in many cases their performance is competitive with complex supervised approaches [16]. Theoretical justifications have been offered for their success [23]. [17] provides a comprehensive overview.

One of the simplest similarity heuristics for networks with a single type of interaction is Common Neighbors, which scores pairs of nodes as likely corresponding to missing links proportional to number of common neighbors those nodes share [18]. Let $\mathcal{G}=$ $\langle\mathcal{V}, \mathcal{E}\rangle$ be a network consisting of undirected edges or links $\mathcal{E}$ which connect distinct nodes in $\mathcal{V}$. For any $v \in \mathcal{V}$, let $\mathcal{N}(v)$ denote the neighbors of $v$ in $\mathcal{G}$, or nodes in $\mathcal{G}$ which are connected to $v$ by an edge in $\mathcal{E}$. Then the Common Neighbors heuristic is defined for distinct nodes $v, v^{\prime} \in \mathcal{V}$ as follows:

$$
\text { CommonNeighbors }\left(v, v^{\prime}\right)=\left|\mathcal{N}(v) \cap \mathcal{N}\left(v^{\prime}\right)\right|
$$

Common Neighbors only considers nodes which are "one-hop" away (neighbors) from the two nodes between which a link is predicted. Another popular "two-hop" heuristic is Adamic-Adar [1]. There are also heuristics which take into account the entire network, as opposed to only a fixed number of hops away, such as the Katz Score [13] and Rooted PageRank [27].

[26] proposes a framework for extending similarity heuristics for link prediction to multiplex networks. We can formally represent a multiplex network as a tuple $\mathcal{G}=\langle\mathcal{V}, \mathcal{E}, \mathcal{T}\rangle$ where $\mathcal{T}$ is a set of edge or link types corresponding to different types of connections between nodes and each edge in $\mathcal{E}$ is between distinct nodes $v, v^{\prime} \in \mathcal{V}$ and of a type $t \in \mathcal{T}$. An equivalent representation is a set of networks which each correspond to a different type of interaction and share the same node sets: $\mathcal{G}=\left\langle\mathcal{G}^{1}, \ldots, \mathcal{G}^{m}\right\rangle$. This later representation is intuitively appealing as the different types of interactions are often described and represented as different layers.

The framework proposed in [26] combines a notion of correlation between layers of multiplex networks with a local feature evaluated at individuals layers. This approach is motivated by the empirical observation that many real world complex systems which can be organized as multiplex networks have highly structured patterns of similarity across layers [19]. Using correlation between layers, it attempts to locate and make use of redundant information from other layers to improve predictions. To construct a measure of crosslayer correlation, first a property matrix is defined which consists of structural features of the network (columns) defined across layers (rows). The structural features most commonly used are shared edges or shared hub nodes, but higher order features, e.g. triangles, etc., can also be used. For example, the property matrix below based on shared edges contains values of 0 or 1 depending on whether a particular edge is present in a given layer:

$$
\mathbf{P}=\begin{gathered}
X-Y \\
\text { Layer 1 } \\
\text { Layer 2 } \\
\text { Layer 3 }
\end{gathered}\left[\begin{array}{ccccc}
1 & 1 & 1 & \ldots \\
1 & 1 & 0 & \ldots \\
1 & 0 & 0 & \ldots
\end{array}\right.
$$

A cross-layer correlation matrix $C$ can then be constructed by applying a correlation metric, e.g. Pearson, Spearman, to all pairwise combinations of the rows of a property matrix, e.g. using $\mathbf{P}$ above, the cross-layer correlation of layers 1 and $2, C_{12}$, is simply the correlations of the vectors corresponding to the first two rows in $\mathbf{P}$.

Using the framework in [26], a heuristic $h$ for link prediction in a multiplex networks is then defined in terms of the cross-layer correlations between two layers $k$ and $k^{\prime}, \rho_{k, k^{\prime}}$, and a local feature which can be considered with respect to any layer, $\phi$ :

$$
h_{i j}^{k}=\frac{1}{Z} \sum_{k^{\prime}} f\left(\rho_{k, k^{\prime}}, \phi_{i j}^{k^{\prime}}\right)
$$

Here, $i$ and $j$ denote the nodes for which a link is predicted at a layer $k$ and $Z$ is a normalizing constant.

The simplest heuristic proposed in [26] is Count and Weight by Correlation (CWC), which simply counts the layers which contain a link between two nodes and weights each count according to its cross-layer correlation with the layer at which predictions are made. Let $A^{k}$ be the adjacency matrix for layer $k$ in a multiplex network, i.e. $A_{i j}^{k}=1$ whenever there is a link between nodes $i$ and $j$ at layer $k$ and $A_{i j}^{k}=0$ otherwise. Then $C W C$ is defined as follows:

$$
C W C_{i j}^{k}=\frac{1}{Z} \sum_{l \neq k} \begin{cases}A_{i j}^{l} \rho_{k, l}, & \rho_{k, l}>0 \\ \left(1-A_{i j}^{l}\right)\left|\rho_{k, l}\right|, & \rho_{k, l}<0\end{cases}
$$

CWC accounts for both positively and negatively correlated layers: if a layer is negatively correlated, the absence of a link at that layer increases the likelihood a link is present at the predicted layer.

While [26] proposes other heuristics for link prediction in multiplex networks, we focus on CWC as its simplicity and structure allows us to efficiently leverage it for the recommendation system we propose. The number of possible links in a multiplex network is quadratic in the number of nodes and linear in the number of layers. It is thus, in general, computationally prohibitive in a real-world setting to calculate a heuristic for all possible node pairs and layers and form a ranking to make recommendations; for large multiplex networks, it is computationally prohibitive even in an offline setting. The structure of CWC, however, allows us to construct a ranking by iterating over the edges present in each layer and filling in the nonzero CWC values using a dynamic programming type of approach. 


\begin{tabular}{c|c|c|c|c|c} 
Network & Layers & Nodes & Max. Layer Correlation & Observed Entropy & Random Network Entropy \\
\hline Pierre Auger Physics Collaboration & 16 & 514 & 0.5308 & 0.002383 & 0.009380 \\
European Airlines & 37 & 450 & 0.3871 & 0.002065 & 0.003398 \\
UN FAO Global Trade & 364 & 214 & 0.7375 & 0.07429 & 0.2930 \\
Yeast Gene-Protein Interactions & 7 & 4092 & 0.1256 & 0.001988 & 0.004792 \\
Financial (Private) & 47 & 530 & 0.6785 & 0.002181 & 0.002511
\end{tabular}

Table 1: Network entropy for real world multiplex networks and comparable randomly generated multiplex networks

This structure also allows us to update these values efficiently using feedback from previous recommendations rather than recomputing the ranking from scratch at each iteration, as we show in section 4 . Furthermore, its simplicity allows for interpretable explanations of its predictions as we discuss in section 4.5 .

\section{MULTIPLEX NETWORK ENTROPY}

Since CWC makes predictions about missing links at a given layer of a multiplex network based on the existence of links in other layers and the similarity between layers, it can be interpreted as enforcing a notion of consistency or order across the network. We can thus use it to construct a notion of entropy for a multiplex network which can then be used to evaluate whether a given set of changes to the structure of the network, i.e. adding or removing edges, results in a lower entropy state. This allows us to formulate the problem of recommending missing and suspicious links in a multiplex network as finding the set of changes to the network which minimizes entropy. While CWC was proposed in [26] for predicting missing links, it can be used similarly for predicting whether existing links are suspicious or anomalous: if CWC is evaluated with respect to existing links in a multiplex network, those which have lower CWC values are most inconsistent with the network and thus most likely to be incorrect or anomalous.

First, we define entropy for existing or possible links in a network as the cross entropy of the reference distribution given by the existence of the link with a distribution estimated using CWC.

Definition 1. In a multiplex network $\mathcal{G}=\left\langle\mathcal{G}^{1}, \ldots, \mathcal{G}^{m}\right\rangle$ with layer adjacency matrices $A^{1}, \ldots A^{m}$, the link entropy, $H_{i j}^{\mathcal{G}^{k}}$, of a (possible) link between nodes $i$ and $j$ at a layer $k$ is the cross entropy of the reference distribution $p(x)$ defined by the existence of the link in $\mathcal{G}$, i.e. the value of $A_{i j}^{k}$, using an estimated distribution $q(x)$ defined by the CWC heuristic applied to that (possible) link:

$$
\begin{aligned}
H_{i j}^{\mathcal{G}^{k}} & =-\sum_{x} p(x) \log q(x) \\
& =-p(0) \log q(0)-p(1) \log q(1) \\
& =-\left[A_{i j}^{k}=0\right] \log \left(1-C W C_{i j}^{k}\right)-\left[A_{i j}^{k}=1\right] \log \left(C W C_{i j}^{k}\right)
\end{aligned}
$$

We then define entropy for a layer as its average link entropy and for a network as its average layer entropy.
Definition 2. In a multiplex network $\mathcal{G}=\left\langle\mathcal{G}^{1}, \ldots, \mathcal{G}^{m}\right\rangle$, the layer entropy of a layer $k$ is its average link entropy:

$$
H^{\mathcal{G}^{k}}=\frac{2}{n(n-1)} \sum_{i \neq j} H_{i j}^{\mathcal{G}^{k}}
$$

Definition 3. In a multiplex network $\mathcal{G}=\left\langle\mathcal{G}^{1}, \ldots, \mathcal{G}^{m}\right\rangle$, the network entropy of $\mathcal{G}$ is its average layer entropy:

$$
H_{\mathcal{G}}=\frac{1}{m} \sum_{k} H^{\mathcal{G}^{k}}=\frac{2}{m n(n-1)} \sum_{k} \sum_{i \neq j} H_{i j}^{\mathcal{G}^{k}}
$$

We expect lower network entropy values for multiplex networks with significant cross-layer correlations. To confirm this we calculate the network entropy of several real-world multiplex networks and compare their network entropy values to those of randomly generated multiplex networks with the same numbers of nodes and layers and the same number of links at each layer: the PierreAuger scientific collaboration network where layers correspond to different collaboration tasks among scientists at the Pierre Auger Observatory, the largest observatory of ultra-high-energy cosmic rays [7], an airline transportation network where layers correspond to direct routes flown by different European airline carriers [6], a global trade network from the United Nations Food and Agriculture Organization where layers correspond to import/export relations among countries for different food items [8], a genetic and protein interaction network for a yeast species where layers correspond to different types of interactions [24] and a private financial network where we expect strong correlations between layers, but also many missing links and possibly some incorrect or suspicious links. We use the Erdos-Renyi random graph model [11] to generate layers with uniformly random links. We report these network entropy values along with the maximum correlation between any two layers in Table 1. As expected, we see lower network entropy for each real-world network than the corresponding randomly generated network. We also observe that the while the private financial network has lower entropy than a random network, the percentage difference is not as significant which is consistent with the knowledge that there are missing and potentially false links in this network. We use this notion of entropy to formalize the problem of recommending missing and false links in the next section.

\section{RECOMMENDATION SYSTEM}

Recommending missing and suspicious links in a multiplex financial network, such as a network of different forms of financial transactions or a network of different types of data flows between internal 
systems at a financial institution, can be interpreted as discovering a set of changes to the network which enforces consistency across layers, or reduces the disorder of the system depicted by the network. We thus formally propose a recommendation system for missing and suspicious links as a system which repeatedly solves a network entropy minimization problem under constraints which express the practical limitations of evaluating and confirming whether the recommendations are correct in a real world context. Once recommendations are confirmed or rejected, the network can be updated to reflect the feedback and the optimization can be performed again to make additional improved recommendations.

\subsection{Formal Problem Solution}

Let $\mathcal{G}=\left\langle\mathcal{G}^{1}, \ldots, \mathcal{G}^{m}\right\rangle$ be a multiplex network with $n$ nodes, $m$ layers and layer adjacency matrices $A^{1}, \ldots A^{m}$. Let $T^{1}, \ldots, T^{m}$ be $n \times n$ matrices of trusted links in $\mathcal{G}$, i.e. $T_{i j}^{k}=1$ if a link between nodes $i$ and $j$ at layer $k$ is trusted, or assumed certain to exist, and $T_{i j}^{k}=0$ otherwise. Let $B^{1}, \ldots, B^{m}$ be $n \times n$ matrices of blocked links in $\mathcal{G}$, i.e. $B_{i j}^{k}=1$ if a link between nodes $i$ and $j$ at layer $k$ is blocked, or assumed certain not to exist, and $B_{i j}^{k}=0$ otherwise. Let $\phi^{+}$and $\phi^{-}$ denote budgets for the number of links to recommended as missing or suspicious, respectively, which can be practically evaluated and confirmed during a given interval. Below, o denotes the Hadamard or element-wise matrix product and $\mathbf{1}_{n}$ denotes a column vector of size $n$ such that all entries are 1. Our proposed recommendation system repeatedly solves the following optimization:

$$
\underset{A^{1 *}, \ldots, A^{m *}}{\operatorname{argmin}} \frac{2}{m n(n-1)} \sum_{k} \sum_{i \neq j} H_{i j}^{\mathcal{G}^{k *}} \text { such that }
$$

(1) $\forall i, j, k, A_{i j}^{k *} \in\{0,1\}$

(2) $\forall i, j, k, A_{i j}^{k *}=A_{j i}^{k *}$

(3) $\sum_{k=1}^{m} \mathbf{1}_{n}^{\top}\left(A^{k *} \circ T^{k}\right) \mathbf{1}_{n}=\sum_{k=1}^{m}\left(\mathbf{1}_{n}^{\top} T^{k} \mathbf{1}_{n}\right)$

(4) $\sum_{k=1}^{m} \mathbf{1}_{n}^{\top}\left(A^{k *} \circ B^{k}\right) \mathbf{1}_{n}=0$

(5) $\sum_{k=1}^{m}\left[\mathbf{1}_{n}^{\top}\left(1-\left[\left(1-A^{k}\right) \circ\left(1-A^{k *}\right)\right]\right) \mathbf{1}_{n}-\mathbf{1}_{n}^{\top} A^{k} \mathbf{1}_{n}\right]=\phi^{+}$

(6) $\sum_{k=1}^{m}\left(\mathbf{1}_{n}^{\top} A^{k} \mathbf{1}_{n}-\mathbf{1}_{n}^{\top}\left[A^{k} \circ A^{k *}\right] \mathbf{1}_{n}\right]=\phi^{-}$

The first two constraints ensure $A^{1 *}, \ldots, A^{m *}$ are valid adjacency matrices. The third and forth constraints ensure no trusted links are recommended as suspicious and no blocked links are recommended as missing. The fifth and sixth constraints ensure the number of missing and suspicious links are equal to their budgets, $\phi^{+}$and $\phi^{-}$.

After solving the above optimization, the recommendations are simply the differences between the entries in $A^{1 *}, \ldots, A^{m *}$ and $A^{1}, \ldots, A^{m}$. After these recommendations are made and feedback is received (confirmations or rejections), we update $A^{1}, \ldots, A^{m}$ with confirmed recommendations and update $T^{1}, \ldots, T^{m}$ and $B^{1}, \ldots, B^{m}$ with suspicious and missing link predictions that were rejected.
Solving this problem, however, requires searching over a combinatorial space, which is computationally infeasible even for small networks in an offline setting. We thus propose a greedy approach which exploits the properties and structure of CWC to allow for efficient recommendations and updates. We provide theoretical and empirical justifications for this approach.

\subsection{Greedy Approach}

Since our proposed formulation of network entropy reduces to a sum of log-transformed CWC values for existing links, an obvious choice for a set of links to add are those corresponding to CWC values closest to 1 . This set is not guaranteed to be optimal, however, since adding these links changes cross-layer correlations which in turn affects other CWC values in the summation. Similarly, the set of existing links in the network with CWC values closest to 0 is an obvious choice for links to remove from the network since these contribute most to the summation. We consider choosing links to add and remove which have the highest and lowest CWC-values, respectively, to be a type of greedy approach to minimizing network entropy since they are optimal given the current set of CWC values, i.e. before recomputing $\mathrm{CWC}$ to reflect the changes to the network.

While choosing the largest and smallest values of CWC for given budgets of links to add and remove significantly simplifies the search problem, it is still computationally demanding in general since (i) CWC values must be computed for a set of possible links that is quadratic in the number of nodes and linear in the number of layers in the network and (ii) the CWC calculation for each link is linear in the number of layers and requires the cross-layer correlation matrix to be recomputed each time the network changes. Fortunately the structure of CWC and formulation of network entropy can be exploited so these values can be updated efficiently using a dynamic programming type of approach. Since the numerator of CWC sums over corresponding links in other layers, we only need to compute CWC for a given link when the corresponding link exists in at least one layer. We can thus compute all necessary CWC values by iterating over the number of layers and links which exist in at least one layer. When making updates, we only need to recompute values in the cross-layer correlation matrix which correspond to layers where links were either added or removed.

We provide our efficient approach as Algorithm 1: Greedy Recommendations. Each run of Algorithm 1 corresponds to a set of updates to the adjacency matrices and trusted and blocked edges as well as the previously cached values for CWC and cross-layer correlations. Links to add and remove are scored at lines 7 and 8 . Then, the top scoring links are iteratively recommended given the budgets in lines 12-28. If the recommendations are confirmed, they are added to the network and flagged; otherwise, they are added to the sets of trusted or blocked edges so these recommendations are not made at future iterations. The cross-layer correlation matrix is then updated for layers which have changed at lines 30-32 and the CWC values are updated. Updates to CWC values can be implemented efficiently since we only need to keep track of CWC values which correspond to links which exist in at least one layer and non-zero values for the numerator of CWC values can be stored in a sparse vector while the denominator is the same for all CWC 


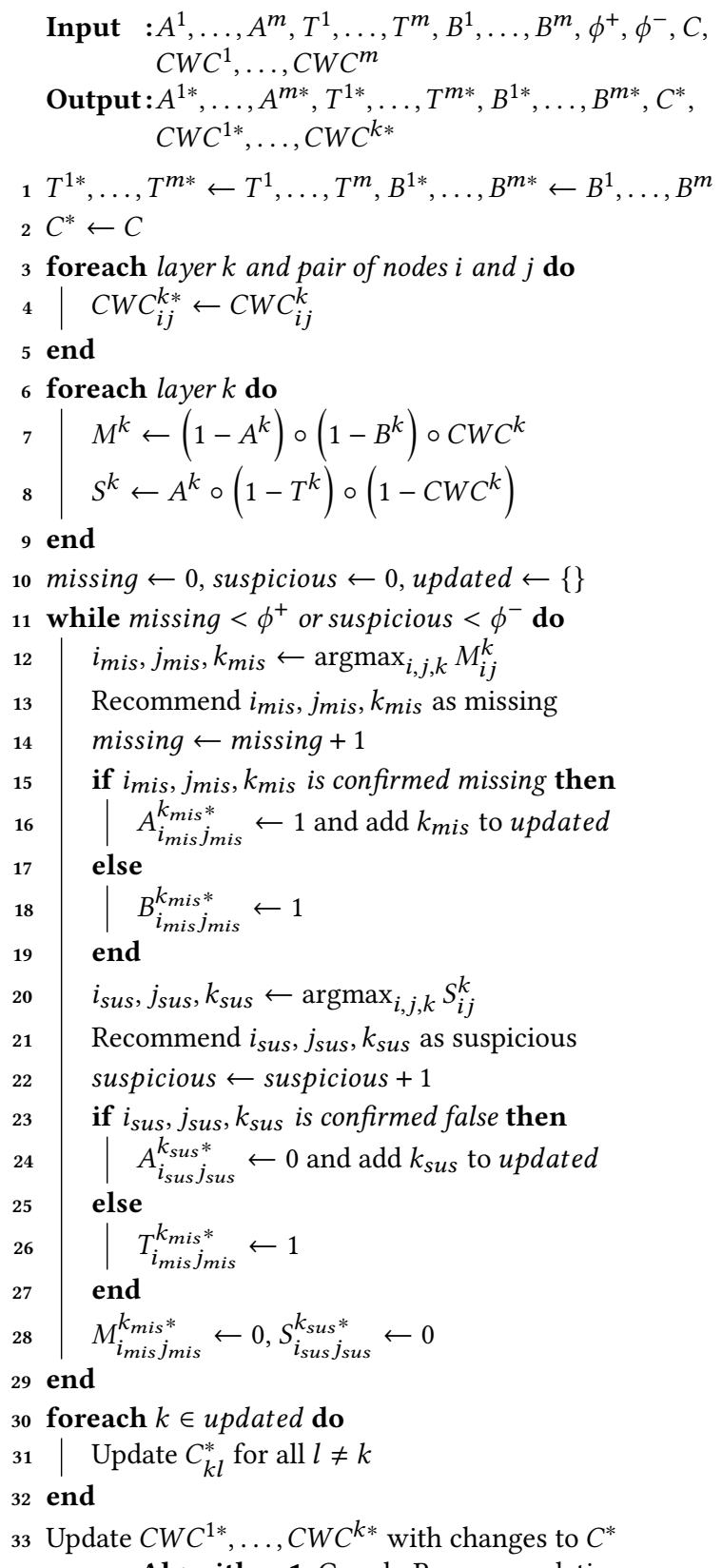

Algorithm 1: Greedy Recommendations

values for a given layer. We now provide theoretical justifications for this greedy approach.

\subsection{Optimality of Greedy Approach}

As discussed above, choosing the highest and lowest CWC values is not guaranteed to result in optimal network entropy since adding and removing links affects the cross-layer correlations used in the CWC calculations for other links. However, it is reasonable to assume that in many cases these recommendations will result in optimal or near-optimal network entropy since they correspond to the largest contributions to the summation in the calculation of network entropy. To theoretically motivate our proposed greedy approach, we identify conditions under which recommending links based on the magnitude of their CWC values is optimal.

Since the numerator of CWC is zero for links which do not exist in any layer, it should never be optimal to recommend such links as missing. It is straightforward to show this.

TheOREm 1. Let $H_{\mathcal{G}+\{u v\}, k}$ represent the network entropy of a multiplex network $\mathcal{G}$ after adding a link between nodes $u$ and $v$ to $\mathcal{G}$ at a layer $k$. If there is no link between nodes $i$ and $j$ in any layer in $\mathcal{G}$ and there is a link between nodes $u$ and $v$ in at least one layer in $\mathcal{G}$ (but not all layers in $\mathcal{G}$ ) then there exists a layer $k$ such that for $1 \leq l \leq m, H_{\mathcal{G}+\{u v\}, k} \leq H_{\mathcal{G}+\{i j\}, l}$.

Proof. Let $k$ be a layer such that there is no link between nodes $u$ and $v$ in $\mathcal{G}$ and let $l$ be any other layer in the network. Then,

$$
\begin{aligned}
& -\log \left(C W C_{i j}^{l}\right)=-\log (0) \\
& -\log \left(C W C_{u v}^{k}\right)=-\log (x) \text { for some } x>0
\end{aligned}
$$

Since $-\log \left(C W C_{u v}^{k}\right)$ is bounded, $-\log \left(C W C_{i j}^{l}\right)$ is unbounded and these terms are included in the summations for $H_{\mathcal{G}+\{u v\}, k}$ and $H_{\mathcal{G}+\{i j\}, l}$, it follows that for $1 \leq l \leq m, H_{\mathcal{G}+\{u v\}, k} \leq H_{\mathcal{G}+\{i j\}, l}$.

This result justifies limiting the search space for missing links to only links present in least one other layer. The same argument justifies recommending links to remove which are not present in any other layer before recommending any other links.

Now we consider the case of choosing between two links to recommend with non-zero CWC values. While is it is not always true that it is optimal to recommend the potential link with the highest CWC value, we can establish conditions based on the magnitude of the difference between the two CWC values where it is optimal to do so. First, in the following lemma, we observe that network entropy can be efficiently updated when adding a link using the previous correlation values. Let $\mathbf{E}_{k}$ represent the set of links in a layer $k$ of a multiplex network with $m$ layers, $e_{k}$ be the cardinality of $\mathrm{E}_{k}$ and $e=\sum_{i=1}^{m} e_{k}$. Also let $\mathbf{c}_{k}$ be the $k$ th column vector of a cross correlation matrix $C$.

LEMmA 2. Let $\mathcal{G}$ be a multiplex network with adjacency matrices $A_{1}, \ldots, A^{m}$, links $\mathbf{E}_{1}, \ldots, \mathbf{E}_{m}$, cross-layer correlation matrix $C$ and $C W C$ values $C W C^{1}, \ldots, C W C^{m}$. For $\{u, v\} \notin \mathrm{E}_{l}$ let $\mathcal{G}^{*}$ represent the multiplex network resulting from adding a link between $u$ and $v$ at layer $l$ to $\mathcal{G}$. Further, let $C^{*}$ and $C W C^{* 1}, \ldots, C W C^{* m}$ be the updated cross-layer correlation matrix and CWC-values. Then

$$
\begin{aligned}
H_{\mathcal{G}^{*}}= & \sum_{k \neq l} e_{k} \log \left(\mathbf{1}_{m}^{\top} \mathbf{c}_{k}+C_{k l}^{*}-C_{k l}\right)+e_{l} \log \left(\mathbf{1}_{m}^{\top} \mathbf{c}_{l}\right) \\
& -\sum_{k \neq l} \sum_{\{i, j\} \in E^{k}} \log \left(\sum_{p \neq l}^{m} A_{i j}^{p} C_{k p}+A_{i j}^{l} C_{k l}^{*}\right) \\
& \sum_{\{i j\} \in E^{l}} \log \left(A_{i j}^{p} C_{p l}^{*}\right)-\log \left(C W C_{u, v}^{* l}\right)
\end{aligned}
$$

Proof. Since network entropy is a summation over log-CWC values for links which exist in the network, adding a link only results in one additional term in the summation so we can write 
network entropy after adding an additional link as a summation over the links in the original network with the updated cross-layer correlations.

$$
\begin{aligned}
H_{\mathcal{G}^{*}}= & -\sum_{k=1}^{m} \sum_{\{i, j\} \in E^{k}} \log \left(\frac{\sum_{p=1}^{m} A_{i j}^{p} C_{k p}^{*}}{\mathbf{1}_{m}^{\top} \mathbf{c}_{k}^{*}}\right)-\log \left(C W C_{u, v}^{* l}\right) \\
= & \sum_{k=1}^{m} e_{k} \log \left(\mathbf{1}_{m}^{\top} \mathbf{c}_{k}^{*}\right)-\sum_{k=1}^{m} \sum_{\{i, j\} \in E^{k}} \log \left(\sum_{p=1}^{m} A_{i j}^{p} C_{k p}^{*}\right) \\
& -\log \left(C W C_{u, v}^{* l}\right)
\end{aligned}
$$

Since adding a link at layer $l$ only changes correlations with $l$, we can write the above in terms of the original correlations.

$$
\begin{aligned}
H_{\mathcal{G}^{*}}= & \sum_{k \neq l} e_{k} \log \left(\mathbf{1}_{m}^{\top} \mathbf{c}_{k}+C_{k l}^{*}-C_{k l}\right)+e_{l} \log \left(\mathbf{1}_{m}^{\top} \mathbf{c}_{l}\right) \\
& -\sum_{k \neq l} \sum_{\{i, j\} \in E^{k}} \log \left(\sum_{p \neq l}^{m} A_{i j}^{p} C_{k p}+A_{i j}^{l} C_{k l}^{*}\right) \\
& \sum_{\{i j\} \in E^{l}} \log \left(A_{i j}^{p} C_{p l}^{*}\right)-\log \left(C W C_{u, v}^{* l}\right)
\end{aligned}
$$

Using the above formula for network entropy, we can specify a condition under which adding a possible link with a higher CWC value is guaranteed to result in a greater reduction in network entropy than a possible link with a lower CWC value.

THeOREM 3. Let $\mathcal{G}$ be a multiplex network with adjacency matrices $A_{1}, \ldots, A^{m}$, links $\mathbf{E}_{1}, \ldots, \mathbf{E}_{m}$, cross-layer correlation matrix $C$ and $C W C$ values $C W C^{1}, \ldots, C W C^{m}$. For $\{u, v\} \notin \mathbf{E}_{l}$ and $\left\{u^{\prime}, v^{\prime}\right\} \notin \mathbf{E}_{l^{\prime}}$ let $\mathcal{G}^{*}\left(\mathcal{G}^{\star}\right)$ represent the multiplex network resulting from adding a link between $u\left(u^{\prime}\right)$ and $v\left(v^{\prime}\right)$ at layer $l\left(l^{\prime}\right)$ to $\mathcal{G}$. Further, let $C^{*}$ $\left(C^{\star}\right)$ and $C W C^{* 1}\left(C W C^{\star 1}\right), \ldots, C W C^{* m}\left(C W C^{\star m}\right)$ be the updated cross-layer correlation matrix and CWC-values after adding these links. Now, assume the magnitude of change in cross-layer correlation values from adding either of these links is at most $\epsilon$ and also assume the minimum correlation between any two layers in the network is $\delta$. If $C W C_{u v}^{* l}>C W C_{u^{\prime} v^{\prime}}^{\star l^{\prime}}$ and the following condition is satisfied, then $H_{\mathcal{G}^{*}} \leq H_{\mathcal{G}^{*}}$.

$$
\log \left(\frac{C W C_{u v}^{* l}}{C W C_{u^{\prime} v^{\prime}}^{\star l^{\prime}}}\right) \geq\left(e-e_{l}-e_{l^{\prime}}\right) \log \left(\frac{m \delta^{2}+2 \epsilon \delta(m+1)+4 \epsilon^{2}}{m \delta^{2}}\right)
$$

Proof. Assume $C W C_{u v}^{* l}>C W C_{u^{\prime} v^{\prime}}^{\star l^{\prime}}$ but $H_{\mathcal{G}^{*}}>H_{\mathcal{G}^{*}}$. Then by Lemma 2, we have the following:

$$
\log \left(\frac{C W C_{u v}^{* l}}{C W C_{u^{\prime} v^{\prime}}^{\star l^{\prime}}}\right)<\left(H_{\mathcal{G}^{*}}+\log C W C_{u v}^{* l}\right)-\left(H_{\mathcal{G}^{\star}}+\log C W C_{u^{\prime} v^{\prime}}^{\star l^{\prime}}\right)
$$

$$
\begin{aligned}
= & \sum_{k \notin\left\{l, l^{\prime}\right\}} e_{k} \log \left(\frac{\mathbf{1}_{m}^{\top} \mathbf{c}_{k}-c_{k l}+c_{k l}^{*}}{\mathbf{1}_{m}^{\top} \mathbf{c}_{k}-c_{k l^{\prime}}+c_{k l^{\prime}}^{\star}}\right) \\
& +e_{l} \log \left(\frac{\mathbf{1}_{m}^{\top} \mathbf{c}_{l}^{*}}{\mathbf{1}_{m}^{\top} \mathbf{c}_{l}^{\star}}\right)+e_{l^{\prime}} \log \left(\frac{\mathbf{1}_{m}^{\top} \mathbf{c}_{l^{\prime}}^{*}}{\mathbf{1}_{m}^{\top} \mathbf{c}_{l^{\prime}}^{\star}}\right) \\
& -\sum_{k \notin\left\{l, l^{\prime}\right\},\{i, j\} \in \mathbf{E}^{k}} \log \left(\frac{\sum_{p \notin\left\{l, l^{\prime}\right\}} A_{i j}^{p} C_{k p}+A_{i j}^{l} C_{k l}^{* l}+A_{i j}^{l^{\prime}} C_{k l}^{l^{\prime}}}{\sum_{p \notin\left\{l, l^{\prime}\right\}} A_{i j}^{p} C_{k p}+A_{i j}^{l} C_{k l}^{l}+A_{i j}^{l^{\prime}} C_{k l}^{l \star^{\prime}}}\right) \\
& -\sum_{\{i, j\} \in \mathbf{E}^{l}} \log \left(\frac{\sum_{p=1}^{m} A_{i j}^{p} C_{p l}^{*}}{\sum_{p=1}^{m} A_{i j}^{p} C_{p l}}\right)-\sum_{\{i, j\} \in \mathbf{E}^{l^{\prime}}} \log \left(\frac{\sum_{p=1}^{m} A_{i j}^{p} C_{p l^{\prime}}}{\sum_{p=1}^{m} A_{i j}^{p} C_{p l^{\prime}}^{\star}}\right) \\
\leq & \sum_{k \notin\left\{l, l^{\prime}\right\}} e_{k} \log \left(\frac{m \delta+2 \epsilon}{m \delta}\right)+\left(e_{l}+e_{l^{\prime}}\right) \log \left(\frac{\delta+\epsilon}{\delta}\right) \\
& -\sum_{k \notin\left\{l, l^{\prime}\right\}} e_{k} \log \left(\frac{\delta}{\delta+2 \epsilon}\right)-\left(e_{l}+e_{l^{\prime}}\right) \log \left(\frac{\delta}{\delta+\epsilon}\right) \\
\leq & \left(e-e_{l}-e_{l^{\prime}}\right) \log \left(\frac{m \delta^{2}+2 \epsilon \delta(m+1)+4 \epsilon^{2}}{m \delta^{2}}\right)
\end{aligned}
$$

We expect that, especially for networks with many nodes and edges, adding a single link will not result in very large changes to crosslayer correlations. This theorem shows us that for sufficiently small possible changes to cross-layer correlations, adding possible links with higher CWC values are guaranteed to result in lower network entropy than adding possible links with lower CWC values. This provides a further theoretical justification for our proposed greedy approach. In the next section, we empirically evaluate this approach.

\subsection{Incorporating Feedback}

We can also improve the performance of our proposed recommendation system by taking advantage of the feedback we receive at each iteration, i.e. confirmed and rejected recommendations. While the predictions made by CWC will become more accurate as we make more recommendations simply because more of the true network will be learned, it may be less accurate for certain layers or specific links or nodes initially, particularly if the links which are missing or incorrect in the network occur in a non-random pattern. We can thus improve our recommendations by decreasing the weight given to predictions which are similar to links, layers or nodes which were previously rejected when recommended. Let $a c c^{k}, a c c_{i}$ and $a c c_{i j}$ correspond to the accuracy of previous recommendations for layer $k$, node $i$ and links between $i$ and $j$. Similarly let $a \bar{c} c$ be the mean (or median) of one of these accuracy measures (taken over layers, nodes or links). A simple rule to use feedback to improve recommendations based on layer accuracy is to update the matrix $M^{k}$ resulting from line 7 of Algorithm 1 as follows, for some $\alpha \geq 1$ :

$$
M^{k} \leftarrow M^{k} \min \left(\frac{a c c_{k}}{a \bar{c} c}, 1\right)^{\alpha}
$$

We can make similar updates for specific nodes or links. We evaluate the effectiveness of this strategy in the next section. 


\subsection{Explainable Recommendations}

Another advantage of using CWC as a basis for recommending missing and anomalous links is that it is interpretable, allowing for explainable recommendations. If a user wishes to understand why a particular missing link recommendation is made, a ranked list of the correlations used in the numerator of CWC for that link can be provided along with their magnitudes. The explanation is then simply that the link is recommended because it exists in each of the listed highly correlated layers and the magnitudes indicate how much each layer contributes to the prediction. The explanation for anomalous links is similar: the link only exists in the provided layers which are not strongly correlated. Such explanations can aid a user attempting to validate whether a recommendation is correct.

\section{EXPERIMENTS}

We empirically evaluated our recommendation system on a private real multiplex financial network with 37 layers, 530 nodes, and 10,944 links across all layers. Each layer corresponds to a different type of data interaction between the entities in the network.

First to confirm our greedy approach selects optimal links from the perspective of minimizing network entropy, we considered adding each link corresponding to the top $500 \mathrm{CWC}$ values closest to 1 . We calculated network entropy after after adding each of these links separately and found the resulting network entropy values have Spearman (rank-based) correlation to the CWC values of $\rho=-0.50$, with $p$-value $3.1 e^{-14}$. This indicates high CWC values are strongly associated with lower resulting network entropy.

Next, we considered the effectiveness of the recommendation system in recovering missing and false links. Since no ground truth exists regarding which links are currently missing or false, we evaluate the effectiveness of our approach by running simulations where we either remove existing links or add additional links and confirm the recommendation system is able to recover these modifications to the network. We do this for different experimental settings where we choose the number of links to remove or add as a percentage of existing links and use different budgets for the number of recommendations to make at each iteration. We run our recommendation system for 100 iterations. During each, we calculate the percentage of false or missing links the recommendation system is able to discover as well as the percentage of correct recommendations made at each iteration. We plot these results, averaged over 100 simulations, for recommending missing and false links in Figures 1 and 2. We also consider making recommendations with and without the feedback strategy for layer accuracy described in section 4.4.

We see that under each condition, our recommendation system is able to discover a large percentage of the missing and false links after only a few iterations. The percentage of recommendations that are correct at each iteration gradually decreases as more of the missing or false links are discovered and is highest, approximately $30 \%$, in the setting where the largest percentage of links have been either removed or added to the network. Note that in this setting, the probability that a random recommendation is correct is $1.9 e^{-7}$. We also see that using the strategy described in section 4.4 for incorporating feedback results in a greater number of missing links discovered at earlier iterations. We did not notice a significant difference when using feedback for recommending false links so we do not plot this strategy in Figure 2 . We set $\alpha=1.5$, but do not see significant differences in the results as we vary $\alpha$. We found that the feedback strategy leads to the greatest improvement when done based on layer accuracy as opposed to node or link accuracy.

\section{CONCLUSION}

There are numerous applications of link prediction in financial networks, many of which are multiplex in nature. We proposed an approach based on a greedy solution to minimizing our proposed notion of entropy in multiplex networks. We presented our approach as a recommendation system for the real world context where missing and false link recommendations are provided continuously according to budget limitations.

Our theoretical results motivate our greedy approach and our experiments confirm that the recommendations made are associated with lower network entropy and show that our recommendation system is able to efficiently discover missing and false links in a real world financial multiplex network.

\section{DISCLAIMER}

This paper was prepared for informational purposes by the Artificial Intelligence Research group of JPMorgan Chase \& Co and its affiliates ("J.P. Morgan"), and is not a product of the Research Department of J.P. Morgan. J.P. Morgan makes no representation and warranty whatsoever and disclaims all liability, for the completeness, accuracy or reliability of the information contained herein. This document is not intended as investment research or investment advice, or a recommendation, offer or solicitation for the purchase or sale of any security, financial instrument, financial product or service, or to be used in any way for evaluating the merits of participating in any transaction, and shall not constitute a solicitation under any jurisdiction or to any person, if such solicitation under such jurisdiction or to such person would be unlawful.

\section{REFERENCES}

[1] Lada A. Adamic and Eytan Adar. 2003. Friends and Neighbors on the Web. Social Networks 25, 3 (2003), 211-230.

[2] Ana Babus. 2016. The formation of financial networks. The RAND fournal of Economics 47, 2 (Summer 2016), 239-272.

[3] Albert-László Barabási, Hawoong Jeong, Zoltán Néda, Erzsébet Regan, Andras Schubert, and Tamás Vicsek. 2002. Evolution of the social network of scientific collaborations. Physica A 311, 3 (2002), 590-614.

[4] Marcel Boersma, Sumit Sourab, Lucas Hoogduin, and Drona Kandhai. 2018. Financial statement networks: an application of network theory in audit. Fournal of Network Theory in Finance 4, 4 (Dec. 2018), 59-85.

[5] Guido Caldarelli, Robert M. May, Tarik Roukny, and Joseph E. Stiglitz. 2016. The price of complexity in financial networks. Proceedings of the National Academy of Sciences 113, 36 (Aug. 2016), 10031-10036.

[6] Alessio Cardillo, Jesús Gómez-Gardeñes, Massimiliano Zanin, Miguel Romance, David Papo, Francisco del Pozo, and Stefano Boccaletti. 2013. Emergence of network features from multiplexity. Scientific Reports 3 (2013), 1344

[7] Manlio De Domenico, Andrea Lancichinetti, Alex Arenas, and Martin Rosvall. 2015. Identifying Modular Flows on Multilayer Networks Reveals Highly Overlapping Organization in Interconnected Systems. Physical Review X 5, 1 (2015), $11-27$.

[8] Manlio De Domenico, Vincenzo Nicosia, Alex, Alexandre Arenas, and Vito Latora. 2015. Structural reducibility of multilayer networks. Nature Communications 6 (2015), 6864.

[9] Mert Demirer, Francis X. Diebold, Laura Liu, and Kamil Yilmaz. 2017. Estimating global bank network connectedness. Fournal of Applied Econometrics 33, 1 (July 2017), 1-15.

[10] Anna Maria D'Arcangelis and Giulia Rotundo. 2016. Complex Networks in Finance. Complex Networks and Dynamics 683 (Sept. 2016), 209-235. 

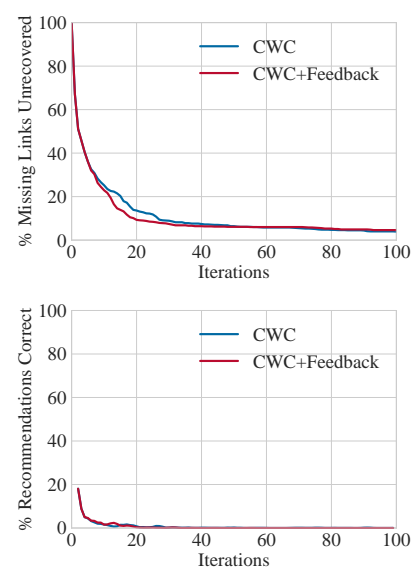

$0.1 \%$ missing, 10 recommendations
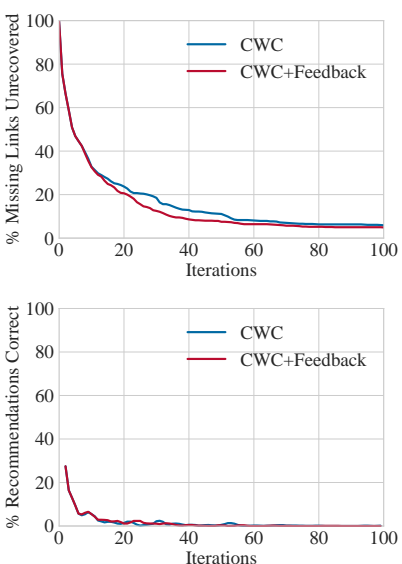

$0.1 \%$ missing, 5 recommendations
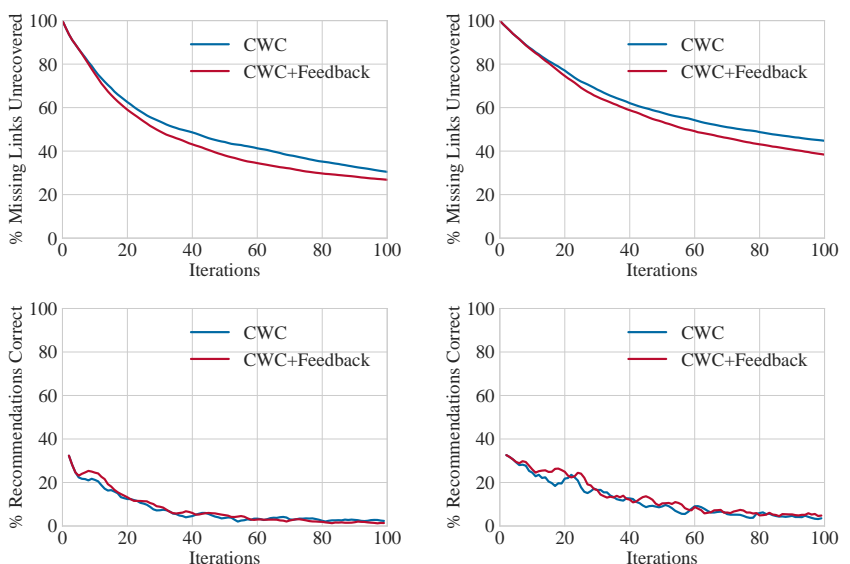

$1 \%$ missing, 10 recommendations

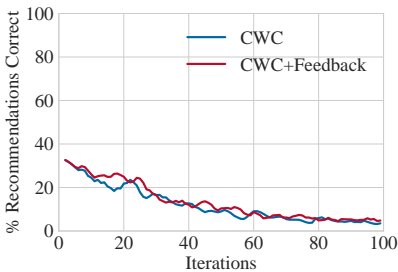

$1 \%$ missing, 5 recommendations

Figure 1: Progress recommending missing links over 100 iterations under different experimental settings
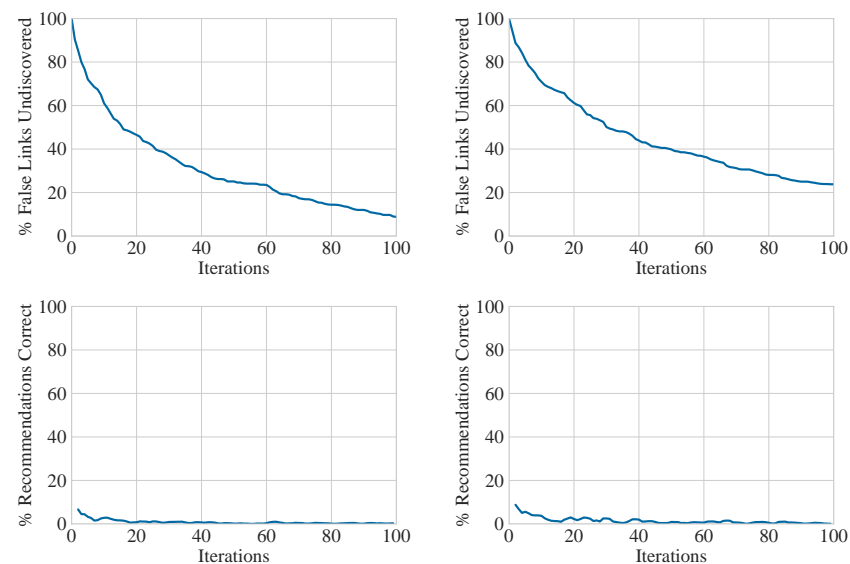

$0.1 \%$ false, 10 recommendations

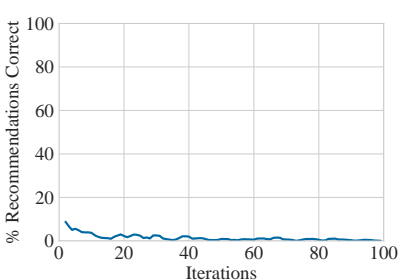

$0.1 \%$ false, 5 recommendations
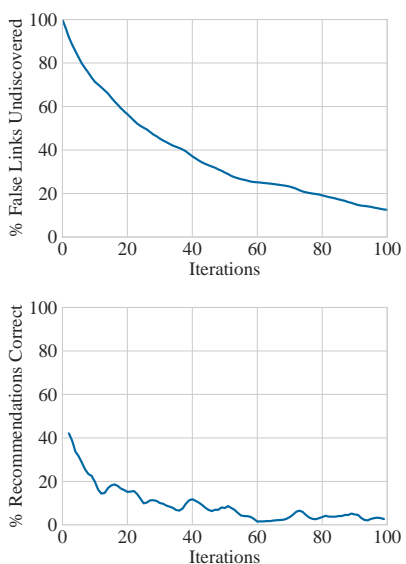

$1 \%$ false, 10 recommendations
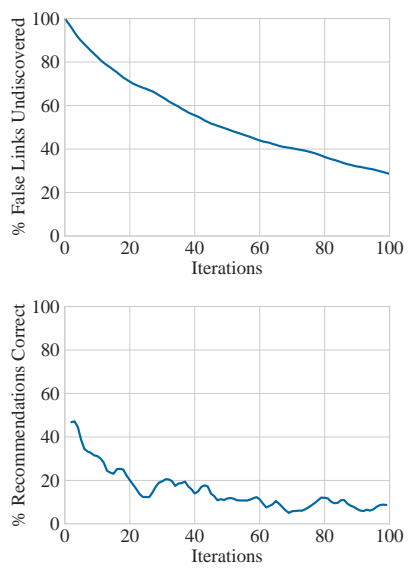

$1 \%$ false, 5 recommendations

Figure 2: Progress recommending false links over 100 iterations under different experimental settings

[11] Paul Erdős and Alfréd Rényi. 1959. On Random Graphs I. Publicationes Mathematicae 6 (1959), 290-297.

[12] Aditya Grover and Jure Leskovec. 2016. node2vec: Scalable Feature Learning for Networks. In Proceedings of the 22nd ACM SIGKDD International Conference on Knowledge Discovery and Data Mining (KDD 2016). ACM, 855-864.

[13] Leo Katz. 1953. A new status index derived from sociometric analysis. Psychometrika 18, 1 (1953), 39-43.

[14] Thomas N. Kipf and Max Welling. 2016. Variational Graph Auto-Encoders. In Proceedings of the NeurIPS 2016 Workshop on Bayesian Deep Learning.

[15] Mikko Kivelä, Alex Arenas, Marc Barthelemy, James P. Gleeson, Yamir Moreno, and Mason A. Porter. 2014. Multilayer Networks. Journal of Complex Networks 2, 3 (2014), 203-271.

[16] David Liben-Nowell and Jon Kleinberg. 2007. The Link-prediction Problem for Social Networks. Journal of the American Society for Information Science and Technology 58, 7 (2007), 1019-1031.

[17] Víctor Martínez, Fernando Berzal, and Juan-Carlos Cubero. 2016. A Survey of Link Prediction in Complex Networks. Comput. Surveys 49, 4 (2016), 69.

[18] ME J Newman. 2001. Clustering and preferential attachment in growing networks. Physical Review E 64, 2 (2001), 025102(R).

[19] Vincenzo Nicosia and Vito Latora. 2015. Measuring and modeling correlations in multiplex networks. Physical Review E 92 (2015).

[20] Matthew Oldham. 2018. The quest for living beta: investigating the implications of shareholder networks. Journal of Network Theory in Finance 4, 2 (June 2018), $25-46$.
[21] Bryan Perozzi, Rami Al-Rfou, and Steven Skiena. 2014. DeepWalk: online learning of social representations.. In Proceedings of the 20th ACM SIGKDD International Conference on Knowledge Discovery and Data Mining (KDD 2014). ACM, 701-710.

[22] Gerard Salton and Michael J. McGill. 1986. Introduction to Modern Information Retrieval. McGraw-Hill, New York.

[23] Purnamrita Sarkar, Deepayan Chakrabarti, and Andrew W. Moore. 2011. Theoretical Justification of Popular Link Prediction Heuristics. In Proceedings of the Twenty-Second International foint Conference on Artificial Intelligence. 2722-2727.

[24] Chris Stark, Bobby-Joe Breitkreutz, Teresa Reguly, Lorrie Boucher, Ashton Breitkreutz, and Mike Tyers. 2006. BioGRID: a general repository for interaction datasets. Nucleic Acids Research 34, 1 (Jan. 2006), 535-539.

[25] Jian Tang, Meng Qu, Mingzhe Wang, Ming Zhang, Jun Yan, and Qiaozhu Mei. 2015. LINE: Large-scale Information Network Embedding. In Proceedings of the 24th International Conference on World Wide Web (WWW 2015). ACM, 1067-1077.

[26] Robert E. Tillman, Vamsi K. Potluru, Jiahao Chen, Prashant Reddy, and Manuela Veloso. 2020. Heuristics for Link Prediction in Multiplex Networks. In Proceedings of the 24th European Conference on Artificial Intelligence (ECAI 2020).

[27] Hanghang Tong, Christos Faloutsos, and Jia-Yu Pan. 2006. Fast Random Walk with Restart and Its Applications. In Proceedings of the Sixth International Conference on Data Mining. 613-622.

[28] Muhan Zhang and Yixin Chen. 2018. Link Prediction Based on Graph Neural Networks. In Advances in Neural Information Processing Systems. Vol. 31. 51655175 . 